\title{
Bee venom acupuncture reduces neuroinflammation modulating microglia/macrophage phenotype polarization in spinal cord injury compression model
}

\author{
Raquel do Nascimento de Souza1, Júlia Miccolis Azevedo Lopes', Lívia da Rocha Natalino Monteiro, \\ Raiana Andrade Quintanilha Barbosa ${ }^{2}$, Gabriela Hollmann ${ }^{2}$, Silvana Allodi ${ }^{2}$, Luis Carlos Reis ${ }^{1}$, Magda \\ Alves de Medeiros ${ }^{1}$
}
'Department of Physiological Sciences, Institute of Biology and Health Sciences, Federal Rural University of Rio de Janeiro, Seropedica 23897-000, RJ, Brazil.
${ }^{2}$ Carlos Chagas Filho Institute of Biophysics, Federal University of Rio de Janeiro, Rio de Janeiro 21941-902, RJ, Brazil.

\begin{abstract}
Correspondence to: Dr. Magda Alves de Medeiros, Department of Physiological Sciences, Institute of Biological and Health Sciences, Federal Rural University of Rio de Janeiro (UFRRJ), BR465, Km 7, Seropedica, 23897-000, RJ, Brazil.

E-mail:magda.medeiros@gmail.com
\end{abstract}

\begin{abstract}
How to cite this article: Souza RN, Lopes JMA, Monteiro LRN, Barbosa RAQ, Hollmann G, Allodi S, Reis LC, Medeiros MA. Bee venom acupuncture reduces neuroinflammation modulating microglia/macrophage phenotype polarization in spinal cord injury compression model. Neuroimmuno/ Neuroinflammation 2019;6:12. http://dx.doi.org/10.20517/2347-8659.2019.04
\end{abstract}

Received: 30 Jul 2019 First Decision: 2 Sep 2019 Revised: 24 Sep 2019 Accepted: 10 Oct 2019 Published: 8 Nov 2019

Science Editor: Athanassios P. Kyritsis Copy Editor: Jin-Wen Zhang Production Editor: Jing Yu

\begin{abstract}
Aim: The present study aimed to examine whether apipuncture (stimulation of acupuncture points with bee venom) at ST36 and GV3 acupoints promotes neuroprotection and reduces neuroinflammation by modulating M1 and M2 phenotype polarization.
\end{abstract}

Methods: Wistar rats were treated with bee venom (BV) $(0.08 \mathrm{mg} / \mathrm{kg})$ injection at acupoints ST36 and GV3 [BV (ST36 + GV3)-spinal cord injury (SCI)] or BV injection at non-acupoints [BV (NP)-SCI] or no treatment (CTL-SCI) after $\mathrm{SCl}$ by compression. The spinal cord mRNA expression of iNOS, Arg-1 and TGF- $\beta$ was measured by real time PCR and the levels of IBA-1; BCL-2; NeuN e CNPase was measured by western blotting. Locomotor performance was measured by Basso, Beattie, and Bresnahan (BBB) and grid-walking tests.

Results: Apipuncture treatment was able to (1) ameliorate locomotor performance; (2) reduce inflammatory markers (Cox-2 levels) and activation of microglia and macrophages; (3) reduce the polarization of the M1 phenotype marker

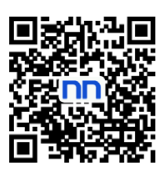


(iNOS) and increase M2 (Arg-1 and TGF- $\beta$ ) phenotypic markers; (4) promote neuroprotection by reducing the death of neurons and oligodendrocytes; and (5) increase the expression of the anti-apoptotic factor BCL-2.

Conclusion: Apipuncture treatment induces locomotor recovery and neuroprotection after the compression model of spinal cord injury. Further, it reduces neuroinflammation by decreasing M1 polarization and increasing M2 phenotype.

Keywords: Acupuncture, bee venom, spinal cord injury compression, microglia, macrophage, neuroinflammation

\section{INTRODUCTION}

Acupuncture, one of the therapies of Traditional Chinese Medicine based on the stimulation of specific body points ${ }^{[1]}$, has shown positive effects in different experimental models of neurodegenerative diseases such as Parkinson's disease (PD) ${ }^{[2]}$, amyotrophic lateral sclerosis (ALS) ${ }^{[3]}$ and spinal cord injury (SCI) ${ }^{[4]}$. Different modalities of acupuncture points (acupoints) stimulation have been used to improve locomotor performance, reduce inflammation and promote neuroprotection in SCI models ${ }^{[4-7]}$. Bee venom (BV) acupuncture (apipuncture) is a therapeutic practice that BV (bee sting itself or diluted) is injected into acupuncture points ${ }^{[8,9]}$.

$\mathrm{BV}$ is a complex substance with approximately 18 bioactive components, including phospholipase A2, histamine, norepinephrine, apamin and melittin (its principal component, representing $40 \%-60 \%$ of the BV total dry extract ${ }^{[9,10]}$. The BV therapy (systemic administration of BV at doses generally between 0.1 and $0.5 \mathrm{mg} / \mathrm{kg}$ ) has anti-nociceptive, anti-tumor, anti-inflammatory and anti-apoptotic effect ${ }^{[9-1]}$. Although several mechanisms of action have been implicated in the therapeutic effects of melittin or the other components of BV, e.g., the anti-arthritis effect of BV has been related to decrease in COX-2 and PLA2 expression and the decrease in the levels of TNF- $\alpha$, IL-1, IL- $6, \mathrm{NO}$, and ROS ${ }^{[9]}$. As BV is a complex substance, various mechanisms of it still need to be revealed.

When BV is applied in acupoints, it promotes intensification of its effects and more intense and lasting acupoint stimulation effects ${ }^{[12,13]}$, which contribute to the clinical improvement of many diseases ${ }^{[9]}$. BV apipuncture has been used to treat inflammatory diseases such as rheumatoid arthritis ${ }^{[14]}$ and to promote pain relief in patients with chronic low back pain ${ }^{[10,15]}$. Besides, it can also be applied as a complementary therapy to treat PD and ALS in humans ${ }^{[10,11,16]}$. Unfortunately, the mechanisms involved in the improvement of nervous system disorders are still poorly understood.

Previous results from our group, using a compression SCI model, showed that apipuncture in ST36 and GV3 acupoints was able to modulate the balance between pro-inflammatory (IL-6) and anti-inflammatory (IL-10) cytokines, promoting a reduction of spinal cord tissue loss and improvement of locomotor performance ${ }^{[17]}$. The neuroinflammation generated after a central nervous system (CNS) traumatic event is one of the most important factors that contribute to neurological losses leading to aggravation of sensory and motor neurological impairment ${ }^{[18-20]}$. However, the inflammatory response also promotes benefits, at certain stages of the inflammatory process, by phagocytizing cellular debris and stimulating tissue repair ${ }^{[18,21,22]}$. The duality of the inflammatory response is believed to be associated with the plasticity of microglia/macrophage polarization status ${ }^{[22-26]}$.

The profile of microglia/macrophage polarization phenotypes in M1/M2 are initially simplified paradigms in an attempt to understand the complexity of the inflammatory respons $\mathrm{e}^{[22,25]}$, in which $\mathrm{M}_{1}$ is described for presenting a pro-inflammatory profile, by stimulating and secreting inflammatory factors such as 
iNOS, IL-1 $\beta$, TNF- $\alpha$; whereas M2 presents anti-inflammatory profile by promoting the release of antiinflammatory cytokines such as IL-10, phagocyte myelin remnants and inhibiting factors to tissue regeneration ${ }^{[18,23,25,27,28]}$. After SCI, there seems to be a dominance of the M1 phenotype ${ }^{[25,27]}$, and although more recent studies have shown that microglia can have multiple activation phenotypes (a "full spectrum of activation") and consider the model of two dualistic microglial state is too simplistic for revision ${ }^{[22]}$; therapeutic approaches that stimulate greater phenotypic polarization of microglia/macrophages in the M2 profile are likely to represent a promising tool ${ }^{[18,23,25]}$.

Thus, in order to expand the previous results, using the same SCI model and followed the same therapeutic methodology, the present study aims to evaluate whether BV injection at ST36 and GV3 acupoints could reduce neuroinflammation by modulating the microglia/macrophages polarization in the M1 and M2 status, reduce apoptosis and promote the neuroprotection of neurons and oligodendrocytes in the SCI model by compression in rats.

\section{METHODS}

\section{Spinal injury compression and groups}

Adult male Wistar rats, weighing between $270 \mathrm{~g}$ and $300 \mathrm{~g}$, were kept in 12/12-h light and dark cycles at a constant temperature, with food and water ad libitum. All procedures were approved by the Ethics Committee on Research of the Federal Rural University of Rio de Janeiro (23083.005880/2013).

Before the surgical procedure, the rats were anesthetized with a mixture of ketamine and xylazine (200 and $10 \mathrm{mg} / \mathrm{kg}$, i.p.; respectively, FortDodge, São Paulo, Brazil). SCI induced by compression was similar to previously described by Vanický et al. ${ }^{[2]]}$. As previously described ${ }^{[17]}$, after exposure of the T10-L1 vertebra, a 2-French Fogarty catheter (Edwards Lifesciences, EUA, CA, USA) was inserted into the epidural space through a small hole in the vertebral arch (mini-laminectomy) of T10 and advanced cranially until the center of the balloon rested at the T8 and T9 level. The balloon catheter was inflated with $15 \mu \mathrm{L}$ of saline for 5 min using a Hamilton syringe (type 1705). A sham-operated group was submitted to a mini-laminectomy without the insertion of the catheter ${ }^{[17]}$.

At the end of the surgical procedure, the animals received injections of analgesic (fentanyl, $0.032 \mathrm{mg} / \mathrm{kg}$, s.c.; Janssen Pharmaceutica, Beerse, Belgium) and prophylactic antibiotic (pentabiotic, 40,000 IU/kg, s.c.; FortDodge, São Paulo, Brazil). The rats with difficulty in spontaneous urination had their bladders emptied manually until they regained voiding function (generally in $12 \mathrm{~h}$ after surgery).

For this study, the animals were randomly divided into 4 groups: (1) Sham group, submitted to the minilaminectomy without the insertion of the catheter; (2) CTL-SCI group which was only submitted to the spinal cord compression; (3) BV (NP)-SCI group received BV at non-acupuncture points at different time points after the SCI; (4) BV (ST36 + GV3)-SCI group received BV injection at ST36 and GV3 acupoints at different time points after the $\mathrm{SCI}^{[17]}$.

\section{BV solution preparation and treatments}

BV (Apis mellifera, catalog \#: V3375; Sigma, St. Louis, MO, USA) at a dose of $0.08 \mathrm{mg} / \mathrm{kg}$ was diluted in saline solution and the application of BV solution was performed according to the group as previously described $^{[17]}$. BV (ST36 + GV3)-SCI group received a subcutaneous injection of BV solution at acupoints ST36 and GV3 ( $20 \mu \mathrm{L}$ at each point). The acupoint ST36 is located approximately $5 \mathrm{~mm}$ below and lateral to the anterior tubercle of the tibia, and GV3 is located on the dorsal midline at the depression between the spinal processes of the last lumbar and the first sacral vertebrae ${ }^{[30]}$. The BV (NP)-SCI group was injected with the same dose and volume of BV at non-acupoints located in the same dermatome as the acupoints. 
For ST36, the non-acupoint was located $5 \mathrm{~mm}$ lateral to the midline of the posterior face of the hindlimb, and for GV3, approximately $1 \mathrm{~cm}$ lateral to the GV3 on the crest of the ilium ${ }^{[17]}$.

Animals subjected to behavioral analysis received BV immediately after the SCI, and then weekly until the fifth week; while rats subjected to methods of spinal tissue extraction (qPCR and Western blotting analyzes) received BV only immediately after SCI. Spinal cord samples were collected at different time points as described in the following topics.

\section{Behavioral analyzes after SCI}

For the evaluation of the locomotor capacity, the animals were submitted to the Basso, Beattie, and Bresnahan (BBB) test as described previously ${ }^{[3]]}$. The BBB test, developed by Basso and colleagues, is a well-established test widely used for investigating the mechanisms involved with the pathophysiology of SCI and possible therapeutic targets. Each animal was placed individually in the center of the open field and was observed for $4 \mathrm{~min}$. During walking in the open field, locomotor parameters are observed and compared with the BBB test scale which has scores ranging from 0 (no spontaneous movement in the hind paws) to 21 (normal locomotion). The evaluation was performed weekly and sometimes up to twice in the same week from the first to the thirty-fifth-day post-injury $(1,4,7,10,14,21,28,35$ days after SCI) by two raters blinded to the experiment. The values were represented as mean \pm standard error.

Based on prior publications ${ }^{[32]}$, a grid walk was constructed for rats using two parallel pieces of acrylic plates (1 $\mathrm{m}$ in length) to hold metal bars $\left(10 \mathrm{~cm}\right.$ in length) with $1-4 \mathrm{~cm} \operatorname{apart}^{[32]}$. Before the injury, rats were trained for 3 days on the apparatus. Each rat was allowed to cross the grid walk 3 consecutive times at 35th day after injury and the number of "footfalls", or the number of times that the animals' hind paws fall through the rungs were counted and represented as mean \pm standard error. Animals unable to move the hind limbs were assigned a maximum of 20 footfalls $^{[4]}$. The grid walk test evaluates the sensorymotor coordination between hindlimbs and forelimbs and examines the deficits in descending motor control $^{[32]}$. Although the BBB is a reliable test, the combined use of other tests as the grid walk can facilitate the distinction of different motor and sensory impairments. Pajoohesh-Ganji et al. ${ }^{[33]}$ have shown that combined scoring method can help the discrimination of different injury levels and produce less variability than the individual tests, which can help to follow motor recovery after SCI.

\section{Western Blotting}

The Western blotting technique was used to evaluate IBA-1; BCL-2; NeuN e CNPase, where approximately $1 \mathrm{~cm}$ of the spinal cord at the lesion site was collected at different time points (days 1, 3, 5 and 7 after the SCI). Initially, the collected tissue was mechanically macerated so that the cellular proteins were fully lysed and homogenized in extraction buffer (Tris- $\mathrm{HCl}, \mathrm{pH}$ 7.2) containing the protease inhibitor cocktail (Protease Inhibitor Cocktail Tablets - Roche Diagnostics, Indianapolis, USA). Immediately after extraction, the samples were centrifuged at $20,000 \mathrm{~g}$ for $40 \mathrm{~min}$ at $4{ }^{\circ} \mathrm{C}$ and the total protein concentration of the supernatant was measured by the BCA method using a spectrophotometer (Thermo Scientific, Washington, DC, USA). To perform the electrophoresis and transfer steps, $30 \mu \mathrm{g}$ of protein per sample were solubilized in a buffered solution [ $20 \%$ glycerol, 1 M Tris ( $\mathrm{pH} 6.8$ ), $4 \%$ sodium dodecyl sulphate (SDS), $0.1 \mathrm{M}$ dithiothreitol and $0.02 \%$ bromophenol blue; $\mathrm{pH}$ 6.8] submitted to SDS-polyacrylamide gel and subsequent transfer of the protein to the nitrocellulose membrane. To promote blocking of non-specific bonds, the membranes remained for $90 \mathrm{~min}$ in blocking solution [10\% BSA dissolved in $1.5 \mathrm{M}$ saline $+0.1 \%$ Tween 20 Tris- $\mathrm{HCl}$ buffer (TBS-T)].

Overnight, the membranes remained incubated with the primary antibodies anti- $\beta$-actin (rabbit, dilution 1:3000, Abcam, Germany), anti-IBA-1 (goat, dilution 1:500, Santa Cruz, USA), anti-GFAP (mouse, dilution 1:1000, Abcam, Germany), anti-BCL-2 (mouse, dilution 1:500, Santa Cruz, USA), anti-NeuN (rabbit, 
dilution 1:1000, Millipore, USA), anti-CNPase (mouse, dilution 1:750, Millipore, USA) at $4{ }^{\circ} \mathrm{C}$ in a solution containing TBS-T and 5\% BSA. After washing they remained incubated with secondary antibodies peroxidase-conjugated (Abcam, Germany) anti-rabbit (dilution 1:5000), or anti-goat (dilution 1:2000), or anti-mouse (dilution 1:1000) diluted in a solution containing 5\% BSA in TBS-T for $1 \mathrm{~h}$.

For detection of the bands, the membrane was incubated with chemiluminescence reagents (ECL; BioRad, Hercules, CA, USA) and suffered 90 s of exposure in ChemiDoc XRS + Imaging System (Bio-Rad, Hercules, CA, USA). $\beta$-actin was applied in the same blot technique for densitometric measurements to normalize the intensities of specific bands using the ImageLab program of Bio-Rad (Bio-Rad, Hercules, CA, USA).

\section{Real-time PCR}

Spinal cord samples from animals of all groups and collected at different time points (days 1, 3, 5 and 7 after the SCI) were submitted to total RNA extraction using QIAzol Lysis Reagent (Qiagen) and eluted in $30 \mu \mathrm{L}$ of RNAse free water. For mRNA analyzes, High Capacity cDNA Reverse Transcription kit (Thermo Fisher Scientific) was used to prepare cDNA in a final concentration of $50 \mathrm{ng} / \mu \mathrm{L}$, following the manufacturer's protocol. For qRT-PCR, the following reaction was prepared: $5.9 \mu \mathrm{L}$ of nuclease-free water, $7.5 \mu \mathrm{L}$ of Power SYBR ${ }^{\oplus}$ Green PCR Master Mix, $10 \mu \mathrm{M}$ of forward and reverse primers and 5 ng of diluted cDNA. Quantitative gene expression was normalized to the expression levels of housekeeping gene $G A P D H$. The target genes from the experimental group were compared with those from the control group using the 2- $\triangle \Delta \mathrm{CT}$ method. Primer sequences for each of the mRNA targets were the following: Arginase-1 forward: 5' ATATCTGCCAAGGACATCGTG 3', reverse: 5' AGGTCTCTTCCATCACTTTGC 3'; iNOS forward: 5' GGAGCAGGTTGAGGATTACTTC 3', reverse: 5' TCAGAGTCTTGTGCCTTTGG 3'; TGF- $\beta$ forward: 5' TGGCGTTACCTTGGTAACC 3', reverse: 5' GGTGTTGAGCCCTTTCCAG 3'; COX-2 forward: 5' TCAAGGGAGTCTGGAACATTG 3', reverse 5' GCTTCCCAACTTTTGTAACCG 3’; GAPDH forward: 5' CCATCAACGACCCCTTCATT 3', reverse: 5' GACCAGCTTCCCATTCTCAG 3'.

\section{Statistical analysis}

All statistical analyses and construction of the graphs were performed by GraphPad Prism 5.0 software (San Diego, CA, USA).To perform the BBB test analysis, the data were submitted to a two-way analysis of variance (ANOVA) for repeated measures followed by Bonferroni post-test. For the analysis of the Grid Walk test, Western blot and qRT-PCR, the data were submitted to one-way ANOVA followed by Bonferroni post-test. For data that did not present normal distribution, the Kruskal Wallis test was performed followed by Dunn's post-test. All dates from this experimental protocol were expressed as mean \pm standard error of the mean. The statistics were considered significant only when $P<0.05$.

\section{RESULTS}

\section{Apipuncture promotes functional recovery in $\mathrm{SCl}$ rats}

After SCI, rats show 0-1 scores in the BBB test, meaning no spontaneous movements in the hind limbs. Compared to CTL-SCI and BV (NP)-SCI groups, BV (ST36 + GV3)-SCI groups showed significant higher scores in BBB test, at 7, 14, 21 and 35 days after SCI (Two way Anova for repeated measures, $P<0.001$ ). In the same way, the BV (ST36 + GV3)-SCI group had a significantly lower footfalls in the grid-walking test in comparison with the CTL-SCI and BV (NP)-SCI groups (One way ANOVA followed by Bonferroni test; $P$ $<0.001$ ) [Figure 1].

\section{Apipuncture changes polarization phenotypes of M1 (iNOS) and M2 (Arg-1 and TGF- $\beta$ )}

BV (ST36 + GV3)-SCI group showed significant lower expression of iNOS mRNA (M1 marker) than CTLSCI and BV (NP)-SCI groups in the 3rd and 5th day after SCI (One way ANOVA followed by Bonferroni 


\section{A}

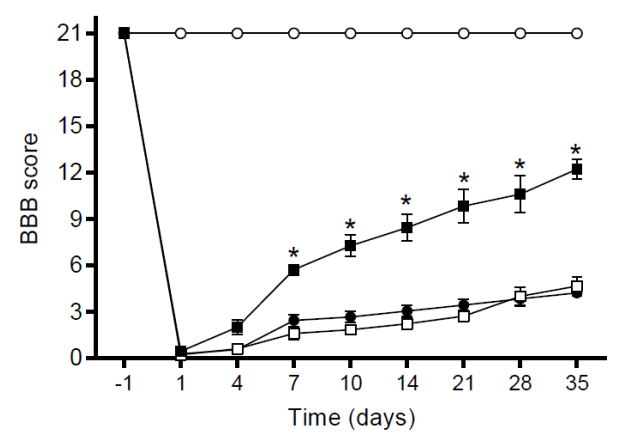

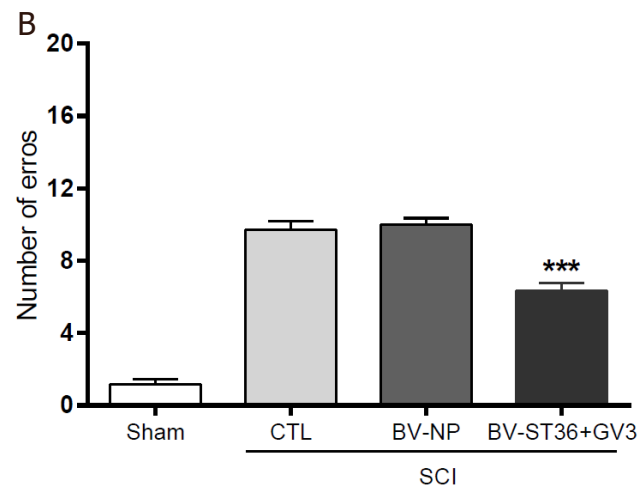

Figure 1. Apipuncture improves locomotor performance in $\mathrm{SCl}$ rats. The results of apipuncture at $\mathrm{GV} 3$ and ST36 acupoints [BV (ST36 + $\mathrm{GV} 3)-\mathrm{SCl}, n=7$ ] were compared to apipuncture at non-acupoints [BV (NP)-SCl, $n=8$ ], control group without manipulation $(\mathrm{CTL}-\mathrm{SCl}, n$ $=7)$ and Sham $(n=7)$ in rats submitted to compression SCI model. BV (ST36 + GV3)-SCI had higher BBB scores $(A)$ at 7, 10, 14, 21, 28 and 25 days after $\mathrm{SCl}$ and lower footfalls in Grid-walking test (B) than CTL-SCI and BV (NP)-SCl groups at 35 days after SCl. Values are presented as mean \pm SEM. ${ }^{\star} P<0.05$ and ${ }^{* \star *} P<0.001$ compared to CTL-SCl and BV (NP)-SCl groups. BV: bee venom; NP: non-acupoints; BBB: Basso, Beattie, and Bresnahan; SCl: spinal cord injury

A

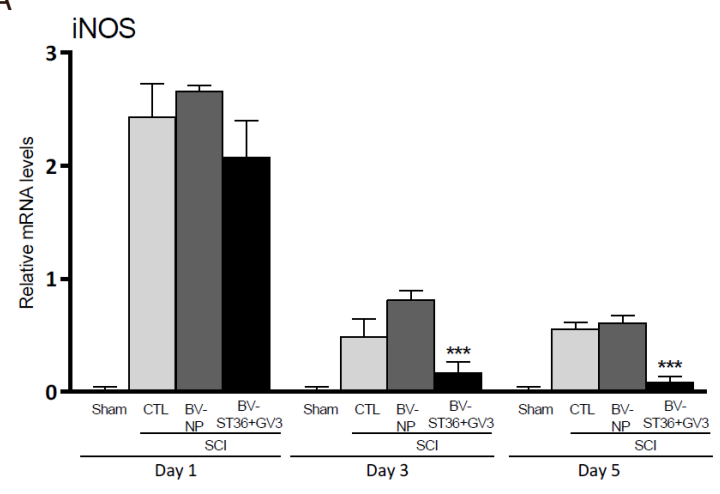

C

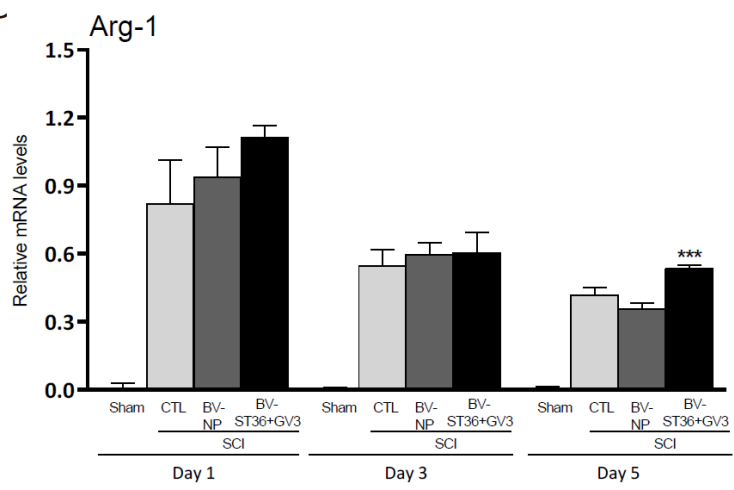

B

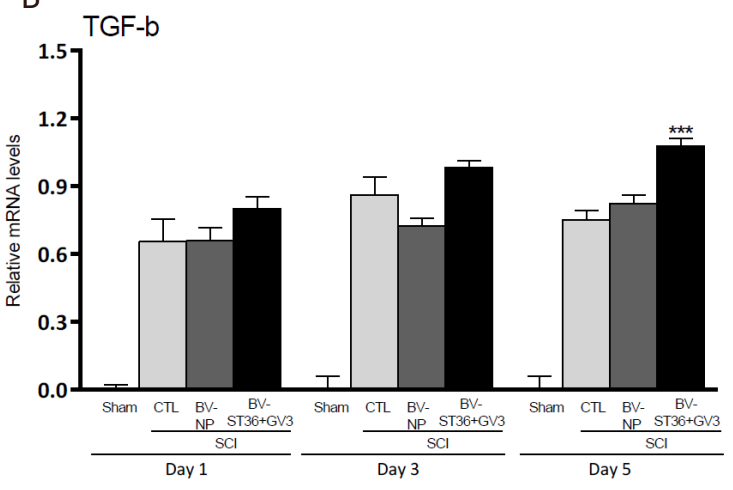

D

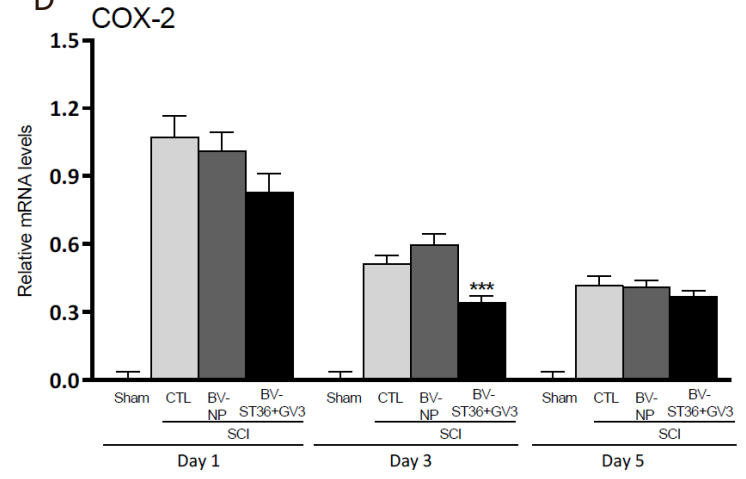

Figure 2. Influence of apipuncture in the mRNA expression of M1 (iNOS) and M2 (TGF- $\beta$ and Arg-1) phenotype markers and COX-2 in the spinal cord 1, 3 and 5 days after SCI. The graph represents the iNOS (A), TGF- $\beta$ (B), Arg- 1 (C) and COX-2 (D) mRNA expression at the site of spinal cord injury in rats submitted to $\mathrm{SCl}$ and apipuncture at $\mathrm{ST} 36$ and $\mathrm{GV} 3$ points [BV (ST36)-SCl; $n=5$ ], $\mathrm{SCl}$ and apipuncture at non-acupoints [BV (NP)-SCl, $n=5$ ], only SCl (CTL-SCl, $n=5)$ and Sham-SCI $(n=5)$. Values are presented as mean $\pm \mathrm{SEM},{ }^{\star \star \star} P<0.001$ compared to CTL-SCl and BV (NP)-SCI. BV: bee venom; NP: non-acupoints; SCl: spinal cord injury

test; $P<0.001$ and $P<0.05$, respectively), and higher expression of Arg-1 and TGF- $\beta$ mRNA (M2 markers) than BV (NP)-SCI and CTL-SCI controls $(P<0.05)$ in the 5th day after SCI [Figure 2]. 

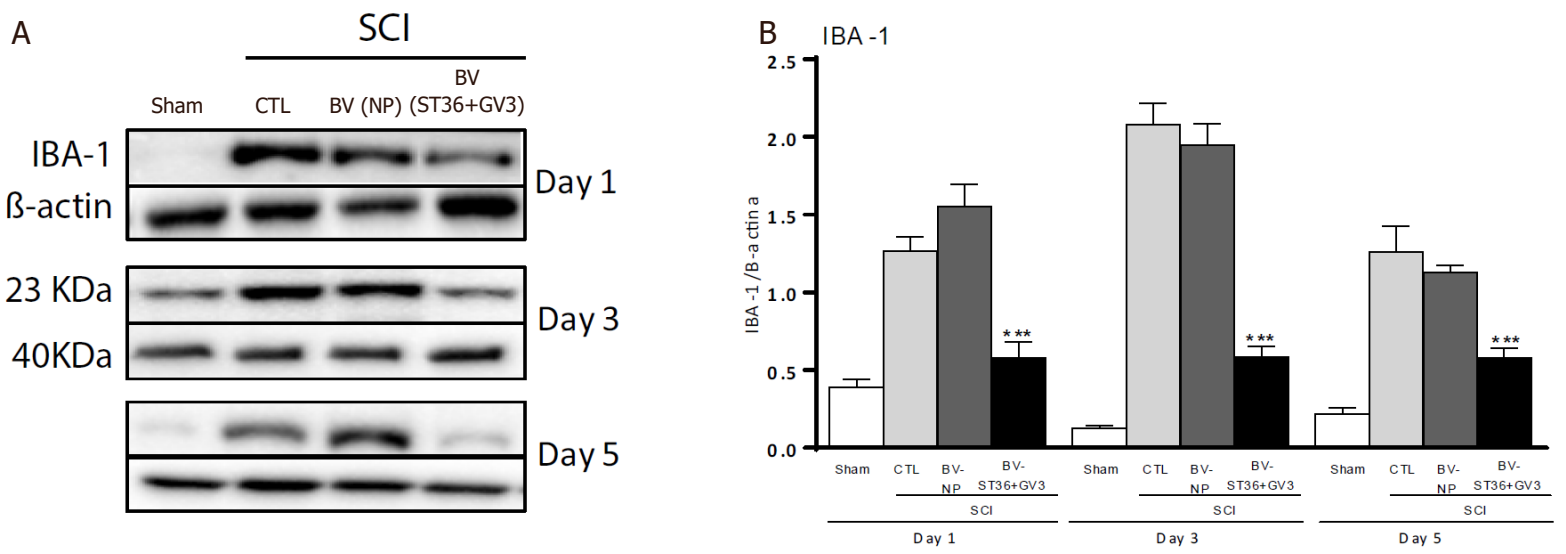

Figure 3. Influence of apipuncture on IBA-1 levels in the spinal cord 1, 3 and 5 days after SCI. In A: representative of the bands of proteins of IBA-1 by the groups Sham, CTL-SCI, BV (NP)-SCI and BV (ST36 + GV3)-SCI measured by densitometry, normalized by $\beta$-actin; In B: the comparison of the IBA-1 protein content at the spinal cord lesion site of rats submitted to SCl and apipuncture at ST36 and GV3 points [BV (ST36)-SCl; $n=6], \mathrm{SCl}$ and apipuncture at non-acupoints [BV (NP)-SCl, $n=6]$, only SCl (CTL-SCl, $n=6)$ and Sham-SCl $(n=6)$. Values are presented as mean \pm SEM. ${ }^{\star \star \star} P<0.001$ compared to $\mathrm{CTL}-\mathrm{SCl}$ and $\mathrm{BV}(\mathrm{NP})-\mathrm{SCl}$. BV: bee venom; NP: non-acupoints; SCl: spinal cord injury

A

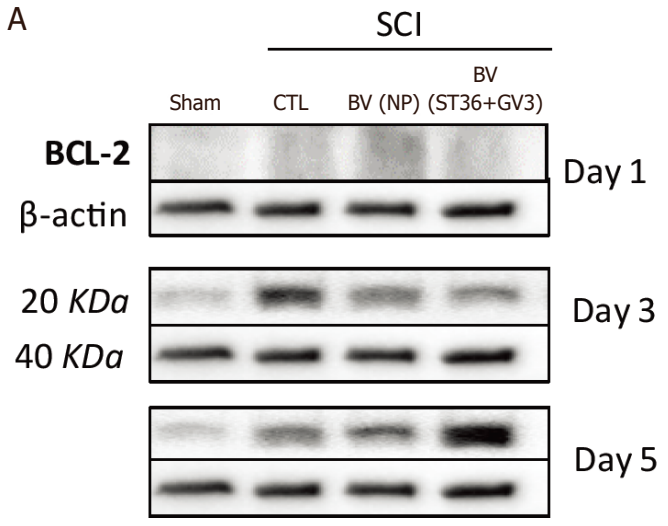

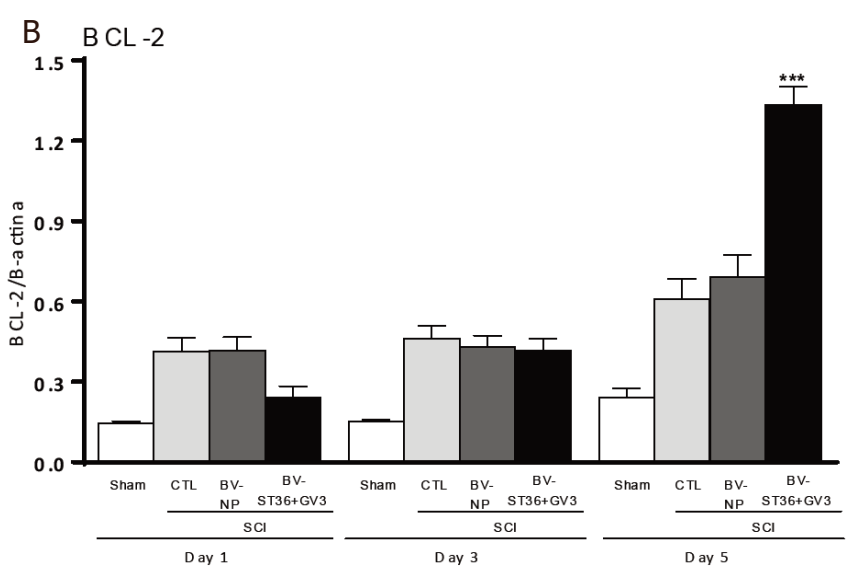

Figure 4. Influence of apipuncture on $\mathrm{BCL}$ levels in the spinal cord 1, 3 and 5 days after $\mathrm{SCl}$. In $\mathrm{A}$ : representative of the bands of proteins of BCL-2 by the groups Sham, CTL-SCI, BV (NP)-SCl and BV (ST36 + GV3)-SCl measured by densitometry, normalized by $\beta$-actin; In B: the comparison of the $\mathrm{BCL}-2$ protein content at the spinal cord lesion site of rats submitted to SCl and apipuncture at ST36 and GV3 points [BV (ST36)-SCl; $n=6], \mathrm{SCl}$ and apipuncture at non-acupoints [BV (NP)-SCl, $n=6]$, only SCl (CTL-SCl, $n=6)$ and Sham-SCl $(n=6)$. Values are presented as mean \pm SEM. ${ }^{\star \star \star} P<0.001$ compared to $\mathrm{CTL}-\mathrm{SCl}$ and $\mathrm{BV}(\mathrm{NP})-\mathrm{SCl}$. BV: bee venom; NP: non-acupoints; SCl: spinal cord injury

\section{Apipuncture reduces activation of microglia/macrophages}

The group BV (ST36 + GV3)-SCI showed significant lower levels of IBA-1 (microglia/macrophage active marker) protein compared to CTL-SCI and BV (NP)-SCI groups at all times evaluated (1st, 3rd and 5th days) after SCI (One way ANOVA followed by Bonferroni test; $P<0.001$ ) [Figure 3].

\section{Apipuncture increases anti-apoptotic factor BCL-2 and neuroprotection of neurons and oligodendrocytes}

The group BV (ST36 + GV3)-SCI showed significant higher levels of BCL-2 (anti-apoptotic factor marker), compared to CTL-SCI and BV (NP)-SCI groups on the 5th day after SCI (One way ANOVA followed by Bonferroni test; $P<0.001$ ) [Figure 4]. Furthermore the group BV (ST36 + GV3)-SCI showed significant higher levels of NeuN (neuron marker) and CNPase protein (enzyme marker expressed by viable oligodendrocytes), compared to CTL-SCI and BV (NP)-SCI groups on the 7th day after SCI (One way ANOVA followed by Bonferroni test; $P<0.001$ ) [Figure 5]. 

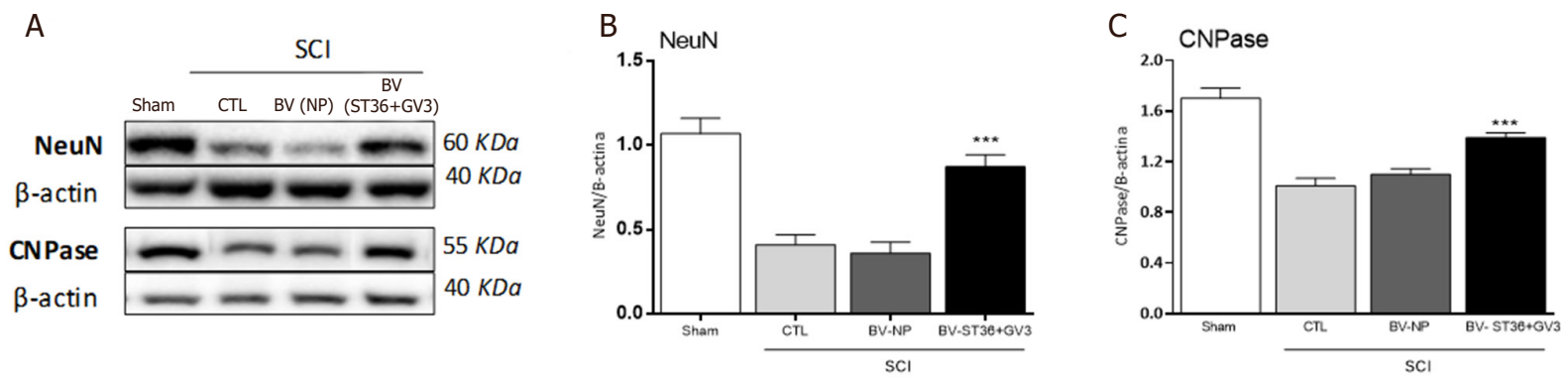

Figure 5. Influence of apipuncture on NeuN and CNPase levels in the spinal cord 7 days after $\mathrm{SCl}$. In A: representative of the bands of proteins of NeuN and CNPase by the groups Sham, CTL-SCI, BV (NP)-SCl and BV (ST36 + GV3)-SCl measured by densitometry, normalized by $\beta$-actin; In B and C: the comparison of the NeuN and anti-CNPase protein content at the spinal cord lesion site of rats submitted to to $\mathrm{SCl}$ and apipuncture at ST36 and $\mathrm{GV} 3$ points [BV (ST36)-SCl; $n=6], \mathrm{SCl}$ and apipuncture at non-acupoints [BV (NP)-SCl, $n=6]$, only SCl $(\mathrm{CTL}-\mathrm{SCl}, n=6)$ and Sham-SCI $(n=6)$. Values are presented as mean $\pm \mathrm{SEM}$. ${ }^{\star \star \star} P<0.001$ compared to $\mathrm{CTL}-\mathrm{SCl}$ and $\mathrm{BV}$ (NP)-SCl. BV: bee venom; NP: non-acupoints; $\mathrm{SCl}$ : spinal cord injury

\section{DISCUSSION}

Our results demonstrated that apipuncture treatment promoted improvement in locomotor function in the SCI compression model in rats. This improvement seems to be associated with neuroinflammation modulation through the reduction of the microglia/macrophage protein marker IBA-1, changes in the expression of M1 (iNOS) and M2 gene markers (TGF- $\beta$ and ARG-1), and decrease of COX-2 expression. Furthermore, apipuncture increased protein levels of the anti-apoptotic marker BCL-2, as well as NeuN and CNPase markers indicating the reduction of the death of neurons and oligodendrocytes at the site of SCI.

The previous study conducted by our group, using the same experimental protocol showed that BV acupuncture at ST36 and GV3 acupoints was able to improve locomotor performance, reduced the area of tissue damage in the spinal cord and modulate the balance between cytokines by increasing the antiinflammatory cytokine IL-10 and reducing the proinflammatory cytokine IL- $6{ }^{[17]}$. From these data, in the present study, we investigate whether the acupuncture mechanism could be associated with M1 and M2 status since apipuncture has shown to influence IBA-1 expression and the balance between proinflammatory and anti-inflammatory cytokine. Our data showed that apipuncture treatment reduced the expression of the iNOS gene a marker associated with M1 status whilst decreased TGF- $\beta$ and ARG-1, associated with M2 status. Zhao et al ${ }^{[7]}$ demonstrated similar results in which electroacupuncture (EA) at Jizhong (GV6) and Zhiyang (GV9) acupoints was able to reduce IL-1 $\beta$, IL-6 and TNF- $\alpha$ proinflammatory cytokines whilst increasing the anti-inflammatory cytokine IL-10. Additionally, EA reduced the proportion of $\mathrm{CD}_{68}{ }^{+}$and $\mathrm{CD} 86^{+}$markers expressed by M1 status and increased $\mathrm{CD} 68^{+}$and $\mathrm{CD}_{206}{ }^{+} \mathrm{M} 2$ markers in immunohistochemical analysis. From these results, the authors suggested that the mitigation of the inflammatory process occurs due to modulation on M1 and M2 status.

In the acute phase of the inflammatory response following SCI, M1 polarization phenotype markers are prevalent and pro-inflammatory factors such as IL1 $\beta$, IL-6, TNF- $\alpha$ and iNOS contributing to positive feedback that triggers an exaggerated inflammatory response causing greater tissue damage around the SCI area ${ }^{[22,25]}$. However, the response generated by M1 status can be modulated by the M2 polarization phenotype that secretes anti-inflammatory factors such as IL-10, IL13, TGF- $\beta$ and Arginase-1 (Arg-1 $)^{[1,27,34]}$.

Previous studies have reported that the use of drugs, such as steroidal anti-inflammatory, that block the acute inflammatory response leads to more neurological damage in the SCI model than when this blockade does not occur ${ }^{[18,20,35]}$. One of the hypotheses for this worsening is intrinsically associated with the complexity of the phenotypic polarization that microglia and macrophages can acquire ${ }^{[18,21,25]}$. In SCI, 
as well as in other neuroinflammatory diseases, there is deregulation between these states of polarization, causing greater death of neurons and oligodendrocytes due to greater initial activation of more cytotoxic status (M1) followed by a later activation of neuroprotective states $\left(\mathrm{M}_{2}\right)^{[18,21,25,26]}$. Thus, it is believed that therapies that promote total blockade of the inflammatory response could inhibit both M1 and M2 status thus hampering tissue repair and aggravating damage after $\mathrm{SCI}^{[20,25]}$. Our data have shown that apipuncture was able to influence the reduction of iNOS, linked to M1 status and the increase of TGF-b and Arg-1, linked to M2 status, thus suggesting that just as EA, apipuncture treatment might modulate inflammation via $\mathrm{M} 1 / \mathrm{M} 2$ status.

After the injury, an inflammatory response is initiated, in which microglia/macrophages quickly become active by chemical signals released by neural cell death ${ }^{[36,37]}$, they are considered as one of the main initiators of the chronic response, triggering greater secondary damage ${ }^{[18,25,34]}$. When active, they express the ionized calcium-binding adapter molecule-1 (IBA-1) which has been widely used as a polarized microglia/macrophage marker ${ }^{[38]}$.

Our data revealed significantly increased IBA-1 levels in the groups submitted to the surgical procedure compared to the sham group in the first $24 \mathrm{~h}$, which remained increased until the 5th day. However, treatment with apipuncture at acupoints ST36 and GV3 significantly reduced the IBA-1 marker compared to control groups. This modulation in the IBA-1 levels is important in the reduction of neuroinflammation since after SCI there is an exacerbated neuroinflammatory response with microglia/macrophages $\operatorname{activation}^{[20,36,39,40]}$, causing greater tissue damage, with the death of neurons and oligodendrocytes in the first hours after the trauma ${ }^{[18,25,41]}$. Similar results were shown by Kang et al ${ }^{[8]}$ using the SCI model followed by apipuncture treatment at ST36 which was able to reduce the IBA-1 marker detected by Western blotting. Moreover, the Kang and colleagues' study, the expression of the IBA-1 marker was very low in the sham group $^{[8]}$. Manual acupuncture at ST36 acupoints can also contribute to the modulation of the inflammatory response due to a reduction of the IBA-1 marker in the ALS model using hSOD ${ }^{\mathrm{G} 93 \mathrm{~A}}$ animals ${ }^{[3]}$. Our results suggest that apipuncture reduces microglia/macrophage polarization, but it is noteworthy that it is likely to be more involved with M1 status as it additionally reduced iNOS mRNA expression. More studies are needed to enhance these results.

In the present study, the apipuncture treatment was able to reduce COX-2 mRNA on the 3rd day after SCI. Although, in some tissues like the brain and the spinal cord COX-2 is constitutively expressed ${ }^{[42]}$ after SCI occurs induction of COX-2 $2^{[4]}$, which increases mainly prostaglandin PGE2 that binds to prostaglandin E receptor subtypes in endothelial cells. It also stimulates the activation of NF- $\mathrm{BB}$ and consequently the nuclear transcription of chemokines such as MIP-1, attracting hematogenous macrophages to the lesion site. It is believed that this mechanism may contribute to a chronic inflammatory response in some situations ${ }^{[42,43]}$. Corroborating with our results, it has been reported that manual acupuncture reduces COX2 expression $24 \mathrm{~h}$ after SCI and that EA also reduces COX-2 expression in the neuropathic pain model ${ }^{[4,44]}$.

In the present study, apipuncture at ST36 + GV3 also increased BCL-2 levels on the 5th after SCI. Additionally, on the 7th day after SCI, apipuncture significantly minimized the reduction of NeuN protein content (a neuron marker) and of CNPase, an enzyme expressed by viable oligodendrocytes, indicating a lower death of these cell types. Previous studies also indicated that acupuncture and electroacupuncture were able to increase BCL-2 protein levels and reduce BAX and caspase-3 levels, maintaining a higher number of viable neurons after $\mathrm{SCI}^{[4,45]}$. After SCI, there is also an imbalance between pro and antiapoptotic proteins. BAX is a pro-apoptotic protein that stimulates mitochondrial damage through the formation of pores in the mitochondrial membrane, releasing cytochrome $\mathrm{C}$ (Cyt-C) which stimulates the cleavage of caspase-3, an enzyme that leads to cell death ${ }^{[46,4]}$. BCL-2 is an important anti-apoptotic protein that acts by modulating BAX and reducing the stimulation of intrinsic apoptotic factors ${ }^{[48,49]}$. Corroborating 
our data, Khalil et al. ${ }^{[50]}$ demonstrated that apipuncture is capable of increasing BCL-2 expression in the PD model.

Despite the limitation of study in not having immunohistochemical analyses, the increase of antiapoptotic factor BCL-2 caused by apipuncture can be related to neuroprotection and survival of neurons and oligodendrocytes resulting in better sensory and locomotor performance. Choi et al ${ }^{[4]}$ demonstrated through the immunohistochemical technique that manual acupuncture in GV26 and GB34 acupoints reduced caspase-3 expression, increased the expression of neuron markers and oligodendrocytes at the lesion site, suggesting that greater survival rate of these cells is related to better performance in the behavioral tests (BBB and Grid Walk test).

The clinical practice of different acupuncture modalities, such as BV acupuncture and EA, has become increasingly used as a complementary therapy for symptom relief in patients with PD, ALS, and SCI ${ }^{[16,51,52]}$. It is noteworthy that in apipuncture, the BV causes a local "irritation" at the acupoints producing a more intense and lasting effect than the simple needling ${ }^{[8,9]}$. In this experimental protocol, the BV was used in a very low dose, with this purpose ${ }^{[12,13]}$. The ST36 and GV3 acupoints were chosen from the combination of other acupoints that were more efficient in promoting the improvement of locomotor behavior in the SCI model (unpublished data).

The exact mechanism involved in the anti-inflammatory effects of acupoint stimulation has not yet been completely elucidated. Currently, it has been postulated the stimulation of acupuncture points acts through the autonomic nervous system, transmitting signals via the vagus nerve and promoting anti-inflammatory responses ${ }^{[53]}$. In experimental models of peripheral inflammation, such as sepsis and rheumatoid arthritis, acupuncture increases vagus nerve activity through a mechanism known as "cholinergic anti-inflammatory reflex ${ }^{,[54]}$. The stimulation of acupoints activates sensory inputs that are propagated by A $\delta$ and $\mathrm{C}$ fibers, resulting in the activation of different brain nuclei involved with peripheral vagal tone regulation and increase of $\mathrm{ACh}$ release ${ }^{[54]}$. In macrophages, ACh down-regulates the NF-Kb pathway by stimulation alpha-7 nicotinic receptor, partially attenuating the inflammatory response. In the present study, the role of vagal activity was not investigated, although it could contribute to the lower macrophage infiltration from the peripheral circulation ${ }^{[6,54]}$. The physiological mechanisms of acupuncture in the SCI models are still uncertain with many questions that need to be clarified. One of the intriguing points about it is that SCI lesion per se does not seem to block the ascending propagation of the neuronal signal produced by acupoint stimulation (once in some studies, including ours, the acupoints used is located below the SCI lesion). Therefore, further studies will be needed to clarify these questions.

In conclusion, despite some limitations, our results indicate that apipuncture may modulate the neuroinflammatory response via alteration of $\mathrm{M} 1 / \mathrm{M} 2$ polarization status, in addition to increasing the apoptotic factor and promoting neuroprotection, which may in part contribute to a reduction in locomotor sequelae in the compression SCI model. Thus, we believe that apipuncture may be a potential therapeutic target as a complementary therapy for the treatment of spinal cord injury.

\section{DECLARATIONS}

\section{Acknowledgments}

We are indebted to Mr. Ipojucan Pereira de Souza for technical assistance.

\section{Authors' contributions}

Made substantial contributions to conception and design of the study: Souza RN, Medeiros MA Performed data analysis and interpretation: Souza RN, Monteiro LRN, Medeiros MA. 
Performed data acquisition, as well as provided administrative, technical, and material support: Souza RN, Lopes JMA, Monteiro LRN, Barbosa RAQ, Hollmann G, Allodi S, Reis LC, Medeiros MA

\section{Availability of data and materials}

Not applicable.

\section{Financial support and sponsorship}

This work was supported by FAPERJ (Research support foundation in the state of Rio de Janeiro) (grand number: 111.616/2010).

\section{Conflicts of interest}

All authors declared that there are no conflicts of interest.

\section{Ethical approval and consent to participate}

All procedures were approved by the Ethics Committee on Research of the Federal Rural University of Rio de Janeiro (23083.005880/2013).

\section{Consent for publication}

Not applicable.

\section{Copyright}

(c) The Author(s) 2019.

\section{REFERENCES}

1. Zhao ZQ. Neural mechanism underlying acupuncture analgesia. Prog Neurobiol 2008;85:355-75.

2. Lin JG, Chen CJ, Yang HB, Chen YH, Hung SY. Electroacupuncture promotes recovery of motor function and reduces dopaminergic neuron degeneration in rodent models of Parkinson's disease. Int J Mol Sci 2017;18:E1846.

3. Yang EJ, Jiang JH, Lee SM, Yang SC, Hwang HS, et al. Bee venom attenuates neuroinflammatory events and extends survival in amyotrophic lateral sclerosis models. J Neuroinflammation 2010;7:69.

4. Choi DC, Lee JY, Moon YJ, Kim SW, Oh TH, et al. Acupuncture-mediated inhibition of inflammation facilitates significant functional recovery after spinal cord injury. Neurobiol Dis 2010;39:272-82.

5. Wei Z, Zhao W, Schachner M. Electroacupuncture restores locomotor functions after mouse spinal cord injury in correlation with reduction of PTEN and p53 expression. Front Mol Neurosci 2018;11:411.

6. Park JY, Namgung U. Electroacupuncture therapy in inflammation regulation: current perspectives. J Inflamm Res 2018;11:227-37.

7. Zhao J, Wang L, Li Y. Electroacupuncture alleviates the inflammatory response via effects on M1 and M2 macrophages after spinal cord injury. Acupunct Med 2017;35:224-30.

8. Kang SY, Roh DH, Choi JW, Ryu Y, Lee JH. Repetitive treatment with diluted bee venom attenuates the induction of below-level neuropathic pain behaviors in a rat spinal cord injury model. Toxins 2015;7:2571-85.

9. Son DJ, Lee JW, Lee YH, Song HS, Lee CK, et al. Therapeutic application of anti-arthritis, pain-releasing, and anti-cancer effects of bee venom and its constituent compounds. Pharmacol Ther 2007;115:246-70.

10. Cherniack EP, Govorushko S. To bee or not to bee: The potential efficacy and safety of bee venom acupuncture in humans. Toxicon 2018;154:74-8.

11. Zhang S, Liu Y, Ye Y, Wang XR, Lin LT, et al. Bee venom therapy: Potential mechanisms and therapeutic applications. Toxicon 2018;148:64-73.

12. Kim HW, Kwon YB, Han HJ, Yang IS, Beitz AJ, et al. Antinociceptive mechanisms associated with diluted bee venom acupuncture (apipuncture) in the rat formalin test: involvement of descending adrenergic and serotonergic pathways. Pharmacol Res 2005;51:183-8.

13. Park HJ, Lee SH, Son DJ, Oh KW, Kim KH, et al. Antiarthritic effect of bee venom: inhibition of inflammation mediator generation by suppression of NF-kappaB through interaction with the p50 subunit. Arthritis Rheum 2004;50:3504-15.

14. Lee JD, Park HJ, Chae Y, Lim S. An overview of bee venom acupuncture in the treatment of arthritis. Evid Based Complement Alternat Med 2005;2:79-84.

15. Seo BK, Lee JH, Sung WS, Song EM, Jo DJ. Bee venom acupuncture for the treatment of chronic low back pain: study protocol for a randomized, double-blinded, sham-controlled trial. Trials 2013;14:16.

16. Ostrovsky DA, Ehrlich A. Bee venom acupuncture in addition to anti-parkinsonian medications may improve activities of daily living and motor symptoms more than medication alone in idiopathic parkinson's disease. Explore (NY) 2019;15:71-3. 
17. Nascimento de Souza R, Silva FK, Alves de Medeiros M. Bee venom acupuncture reduces interleukin-6, increases interleukin-10, and induces locomotor recovery in a model of spinal cord compression. J Acupunct Meridian Stud 2017;10:204-10.

18. DiSabato DJ, Quan N, Godbout JP. Neuroinflammation: the devil is in the details. J Neurochem 2016;139 Suppl 2:136-53.

19. Oyinbo CA. Secondary injury mechanisms in traumatic spinal cord injury: a nugget of this multiply cascade. Acta Neurobiol Exp (Wars) 2011;71:281-99.

20. Esposito E, Cuzzocrea S. Anti-TNF therapy in the injured spinal cord. Trends Pharmacol Sci 2011;32:107-15.

21. Ulndreaj A, Chio JC, Ahuja CS, Fehlings MG. Modulating the immune response in spinal cord injury. Expert Rev Neurother 2016;16:1127-9.

22. Cherry JD, Olschowka JA, O'Banion MK. Neuroinflammation and M2 microglia: the good, the bad, and the inflamed. J Neuroinflammation 2014;11:98.

23. Chen J, Wu Y, Duan FX, Wang SN, Guo XY, et al. Effect of M2 macrophage adoptive transfer on transcriptome profile of injured spinal cords in rats. Exp Biol Med (Maywood) 2019;244:880-92.

24. Ma SF, Chen YJ, Zhang JX, Shen L, Wang R, et al. Adoptive transfer of M2 macrophages promotes locomotor recovery in adult rats after spinal cord injury. Brain Behav Immun 2015;45:157-70.

25. Gensel JC, Zhang B. Macrophage activation and its role in repair and pathology after spinal cord injury. Brain Res 2015;1619:1-11.

26. Ren Y, Young W. Managing inflammation after spinal cord injury through manipulation of macrophage function. Neural Plast 2013;2013:945034.

27. Zhang Y, Liu Z, Zhang W, Wu Q, Zhang Y, et al. Melatonin improves functional recovery in female rats after acute spinal cord injury by modulating polarization of spinal microglial/macrophages. J Neurosci Res 2019;97:733-43.

28. Zhou Y, Li N, Zhu L, Lin Y, Cheng H. The microglial activation profile and associated factors after experimental spinal cord injury in rats. Neuropsychiatr Dis Treat 2018;14:2401-13.

29. Vanický I, Urdzíková L, Saganová K, Čízková D, Gálik J. A simple and reproducible model of spinal cord injury induced by epidural balloon inflation in the rat. J Neurotrauma 2001;18:1399-407.

30. Yin CS, Jeong HS, Park HJ, Baik Y, Yoon MH, et al. A proposed transpositional acupoint system in a mouse and rat model. Res Vet Sci 2008;84:159-65.

31. Basso D, Beattle M, Bresnahan J. Sensitive and reliable locomotor rating scale for open field testing in rats. J Neurotrauma 1995;12:1-21.

32. Metz GA, Merkler D, Dietz V, Schwab ME, Fouad K. Efficient testing of motor function in spinal cord injured rats. Brain Res 2000;883:165-77.

33. Pajoohesh-Ganji A, Byrnes KR, Fatemi G, Faden AI. A combined scoring method to assess behavioral recovery after mouse spinal cord injury. Neurosci Res 2010;67:117-25.

34. Kumar A, Alvarez-Croda DM, Stoica BA, Faden AI, Loane DJ. Microglial/Macrophage polarization dynamics following traumatic brain injury. J Neurotrauma 2016;33:1732-50.

35. Daltaban IS, Misir S, Turksoy VA, Ak H, Cakir E. The effects of barnidipine on an experimental ischemia reperfusion model of spinal cord injury and comparison with methyl prednisolone. North Clin Istanb 2018;6:103-9.

36. Kwon BK, Tetzlaff W, Grauer JN, Beiner J, Vaccaro AR. Pathophysiology and pharmacologic treatment of acute spinal cord injury. Spine J 2004;4:451-64.

37. Hausmann ON. Post-traumatic inflammation following spinal cord injury. Spinal Cord 2003;41:369-78.

38. Hoogland IC, Houbolt C, van Westerloo DJ, van Gool WA, van de Beek D. Systemic inflammation and microglial activation: systematic review of animal experiments. J Neuroinflammation 2015;12:114.

39. Loane DJ, Byrnes KR. Role of microglia in neurotrauma. Neurotherapeutics 2010;7:366-77.

40. Jones TB, McDaniel EE, Popovich PG. Inflammatory-mediated injury and repair in the traumatically injured spinal cord. Curr Pharm Des 2005;11:1223-36.

41. Shechter R, London A, Varol C, Raposo C, Cusimano M, et al. Infiltrating blood-derived macrophages are vital cells playing an antiinflammatory role in recovery from spinal cord injury in mice. PLoS Med 2009;6:e1000113.

42. Aoki T, Narumiya S. Prostaglandins and chronic inflammation. Trends Pharmacol Sci 2012;33:304-11.

43. Wang ZH, Xie YX, Zhang JW, Qiu XH, Cheng AB, et al. Carnosol protects against spinal cord injury through Nrf-2 upregulation. J Recept Signal Transduct Res 2016;36:72-8.

44. Ji LL, Guo MW, Ren XJ, Ge DY, Li GM, et al. Effects of electroacupuncture intervention on expression of cyclooxygenase 2 and microglia in spinal cord in rat model of neuropathic pain. Chin J Integr Med 2017;23:786-92.

45. Liu J, Wu Y. Electro-acupuncture-modulated miR-214 prevents neuronal apoptosis by targeting bax and inhibits sodium channel Nav1.3 expression in rats after spinal cord injury. Biomed Pharmacother 2017;89:1125-35.

46. Liu C, Shi Z, Fan L, Zhang C, Wang K, et al. Resveratrol improves neuron protection and functional recovery in rat model of spinal cord injury. Brain Res 2011;1374:100-9.

47. Genovese T, Esposito E, Mazzon E, Muià C, Di Paola R, et al. Evidence for the role of mitogen-activated protein kinase signaling pathways in the development of spinal cord injury. J Pharmacol Exp Ther 2008;325:100-14.

48. Wu B, Liang J. Pectolinarigenin promotes functional recovery and inhibits apoptosis in rats following spinal cord injuries. Exp Ther Med 2019;17:3877-82.

49. Luo Y, Fu C, Wang Z, Zhang Z, Wang H, et al. Mangiferin attenuates contusive spinal cord injury in rats through the regulation of oxidative stress, inflammation and the Bcl2 and Bax pathway. Mol Med Rep 2015;12:7132-8.

50. Khalil WK, Assaf N, ElShebiney SA, Salem NA. Neuroprotective effects of bee venom acupuncture therapy against rotenone-induced 
oxidative stress and apoptosis. Neurochem Int 2015;80:79-86.

51. Fan Q, Cavus O, Xiong L, Xia Y. Spinal cord injury: how could acupuncture help? J Acupunct Meridian Stud 2018;11:124-32.

52. Sudhakaran P. Amyotrophic lateral sclerosis: an acupuncture approach. Med Acupunct 2017;29:260-8.

53. Lim HD, Kim MH, Lee CY, Namgung U. Anti-inflammatory effects of acupuncture stimulation via the vagus nerve. PLoS One 2016;11:e0151882.

54. Tracey KJ. Physiology and immunology of the cholinergic antiinflammatory pathway. J Clin Invest 2007;117:289-96. 